\title{
Flora Medicinal de la Región Atlántica de Nicaragua
}

\author{
Medicinal Flora of the Atlantic Region of Nicaragua
}

\author{
Felix G. Coe ${ }^{1}$ \\ Universidad de Connecticut
}

\section{RESUMEN}

En este texto son enumeradas las diferentes investigaciones realizadas en torno a las plantas medicinales en Nicaragua; los factores ambientales que ponen en peligro la existencia de este tipo de plantas y cómo la información etnobotánica se está perdiendo a un ritmo aún más rápido que las especies. También la metodología para la recopilación de la bibliografía relevante a los estudios botánicos en Nicaragua; el estudio de ejemplares de herbario en la obtención de datos etnobotánicos, florístico, ecológicos y taxonómico; la identificación de estos ejemplares botánicos, y el trabajo de laboratorio (análisis químico y bioensayos de los ejemplares botánicos). Igualmente se presenta una interesante reflexión sobre etnobotánica y los derechos de propiedad intelectual como un derecho humano fundamental de todos los pueblos indígenas y el peligro que enfrentan por parte de transnacionales y corporaciones. Finalmente muestra cuadros de las especies medicinales nativas, su hábitat de procedencia, el uso por parte de los grupos indígenas y su bioactividad.

Palabras claves: plantas medicinales; etnofarmacologia; botánica; fitoquimica; Nicaraguaregión atlántica

\begin{abstract}
In this tex they are enumerated the different researches carried out around medicinal plants in Nicaragua are enumerated, the environmental factors that endanger the existence of these types of plants and how the ethno botanical information is being lost at an even faster rate than the species. It explains the methodology for: the study of herbarium specimens (obtaining ethno botanical, floristic, ecological and taxonomic data), their identification and also for laboratory work (chemical analysis and bioassays of botanical specimens). It reflects on ethno botany and intellectual property rights as a fundamental human right of all indigenous peoples and the danger they face from transnationals and corporations. At the end it presents pictures of native medicinal species, their habitat of origin, the use by indigenous groups and their bioactivity.
\end{abstract}

Key Words: medicinal plants of the atlantic-region of (nicaragua); etnopharmacology; ethnobotany; phytochemistry; nicaragua-región atlántica

Recibido: 16 de Octubre del 2018

Aceptado: 24 de Noviembre del 2018

${ }^{1} \mathrm{Ph}$ D en Botánica, por la Universidad de Connecticut (1994)

(C) Copyright 2018. Bluefields Indian \& Caribbean University 


\section{INTRODUCCIÓN}

La agricultura sigue siendo el principal factor de la deforestación a nivel mundial. Pese a los avances en muchos lugares del mundo, la pérdida de cobertura forestal sigue siendo la tendencia dominante a nivel global. Los bosques del mundo se han reducido en un 3\% (se han perdido unos 130 millones de hectáreas) en los últimos 25 años (FAO 2016). La dependencia de ser humano en el reino vegetal data de los tiempos prehistóricos. Las plantas son fuentes de innumerables recursos necesarios para nuestra existencia y bienestar. De ellas obtenemos productos alimenticios, cosméticos y fármacos. Existen registros escritos de las antiguas civilizaciones egipcias y chinas que han hecho uso de estos productos. En la actualidad se ha producido un retorno a la búsqueda de productos llamados "naturales", que en realidad nunca dejó de existir. El análisis de la composición de muchos fármacos muestra que casi el 50\% de los que están en uso clínico son derivados de compuestos naturales, la mayoría son de plantas.

El consumo de medicamentos derivados de plantas está muy difundido y se ha aumentado significativamente tanto en la medicina tradicional como en la moderna. De acuerdo con la Organización Mundial de la Salud (OMS), más del 80\% de la población mundial en los países en desarrollo depende principalmente de medicamentos a base de plantas para las necesidades básicas de salud (Canter et al., 2005). Teniendo en cuenta que la mayoría de las personas de la región atlántica de Nicaragua todavía dependen de la medicina tradicional en forma de remedios a base de hierbas como su fuente de atención primaria de salud, es importante documentar la tradición etnomedicinal (etnofarmacolgía) de estas personas, acumuladas durante miles de años e innumerables generaciones en forma de historia oral. El uso de remedios a base de plantas medicinales sigue siendo cuestionable en la mente de muchas personas, por lo tanto, aquí trato de proporcionar una base científica de las propiedades químicas y datos de bioensayos para justificar por qué estas plantas siguen siendo utilizadas para tratar dolencias específicas.

La región atlántica es en muchos aspectos la región más compleja y variada de Nicaragua. Es el hogar de varias etnias: miskita, rama, sumu y garífunas (descendientes de los arawaks y caribes) (Crawford, 1984). La destrucción de los bosques tropicales en la región atlántica de Nicaragua, como en otras partes del mundo, es indiscriminada. Desafortunadamente, la información etnobotánica se está perdiendo a un ritmo aún más rápido que las especies y el hábitat. Hay relativamente poca información etnobotánica de Nicaragua, y en particular, casi nada se ha escrito acerca de la medicina popular.

En este sentido, en una bibliografía anotada de dos volúmenes que cubre la medicina tradicional a nivel mundial, Cosminsky y Harrison (1984) catalogaron 2,500 referencias, cuatro de las cuales se trata de material sobre Nicaragua y ninguna de ellas es de gran importancia. En contraste, casi 100 obras fueron documentadas para Guatemala y casi 400 para México. Enrique Peña Hernández (1968) dio una excelente reseña de las costumbres y tradiciones folclóricas en Nicaragua, pero este tratamiento incluye poca etnobotánica y, más específicamente, nada sobre la región atlántica de Nicaragua. Miranda (1967) publicó un estudio preliminar de la práctica médica tradicional en el oeste de Nicaragua. El médico nicaragüense Dávila Bolaños publicó (1974) una excelente descripción de la medicina indígena prehistórica basada en una vida de 
observaciones entre los descendientes de los matagalpas y nicaraos del centro de Nicaragua. En 1981, Juan B. Salas dio una breve descripción de las plantas medicinales utilizadas en el Departamento de Managua. Los antropólogos Philip Dennis (1981, 1984, 1988), Mary Helms (1971, 1983) y Franklin Loveland (1975a, b, 1976, 1982), los geógrafos culturales W. V. Davidson (1980) y Bernard Nietschmann (1969, 1972), 1973, 1979, 1990), y el médico Bruce Barrett (1994), han publicado sobre los grupos indígenas de la región atlántica de Nicaragua. Dennis (1988) ofrece descripciones de 23 plantas medicinales usadas por los miskitos, Loveland (1975a) describe las curas de mordedura de serpiente entre los ramas, y Barrett (1994) informa sobre antropología médica en la costa atlántica de Nicaragua.

Uno de los primeros trabajos florísticos en que se menciona la flora de Nicaragua fue hecha por William Botting Hemsley (1879-1888); más tarde, Miguel Ramírez Goyena (1909-1911) publicó su Flora Nicaragüense en dos volúmenes; luego, Eddie Salter (1957) publicó De la Flora Nicaragüense, una compilación de información sobre plantas de uso económico del país; más recientemente, Frank Conkling Seymour (1980) publicó su A checklist of the vascular plants of Nicaragua; Davidse G, Sousa M (1995) Flora Mesoamericana, vol. 1; Davidse G, Sousa M

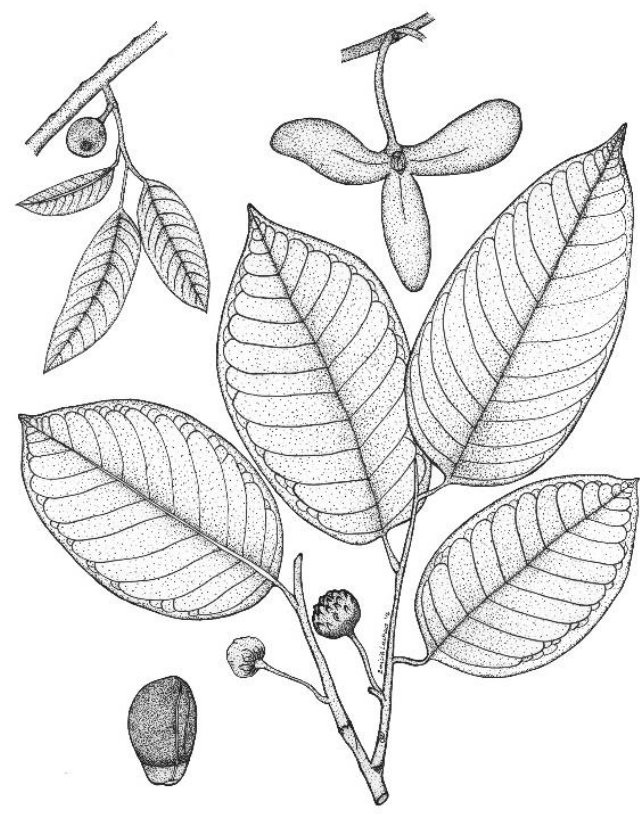
(1995) Flora Mesoamericana, vol. 6; Rico-Arce M, Davidse G, Sousa M, Chater AO (1995) Flora Mesoamericana: Alismataceae to Cyperaceae, vol. 6; Gomez LD, y Arbelaez A (2009). Flora de Nicaragua: Tomo IV, Helechos; Stevens et al. (2001) La Flora de Nicaragua, Tomos I, II, III; Grijalva (2002) Etnobotánica de Nicaragua; Davidse G, Sousa M (2009) Flora Mesoamericana, vol. 4, parte 1; Davidse G, Ulloa C (2012) Flora Mesoamericana, vol. 4, parte 2; y Davidse G, Ulloa C (2015) Flora Mesoamericana, vol. 2, parte 3.

Respecto a estudios etnobotánicas en Nicaragua, éstos eran casi inexistentes antes de 1972. Recientemente, este autor ha contribuido al saber etnobotánico de la región atlántica de Nicaragua (Coe 1994; Coe y Anderson 1996a, 1996b, 1997, 1999, 2005; Coe 2008a, 2008b; Coe, Parikh and Johnson 2010; Coe et al. 2012).

Fig. 1. Annona papilionella. Dibujo de Feliz Coe.

Mis estudios sistemáticos sobre la etnobotánica, fitoquímica y citotoxicidad de las plantas medicinales de la región atlántica de Nicaragua son los primeros de esta índole. Esta información es muy importante debido a la desaparición de muchas de las especies causada por la deforestación indiscriminadas que existe en esta región del país. La documentación del potencial farmacológico de las plantas de esta región servirá a las futuras generaciones como fuente de medicina para la curación de enfermedades y el mantenimiento de la salud de la población en esta región. 
El objetivo de este libro es primero documentar la flora medicinal de la región atlántica de Nicaragua; segundo, determinar las propiedades fitoquímicas y biológicas de las plantas que figuran en este documento y tratar de correlacionar estas propiedades con los usos populares, así como para evaluar si su composición química puede soportar las propiedades biomédicas reportadas como relacionadas con las mismas.

\section{GEOGRAFIA}

Nicaragua es el país más grande de Centroamérica con una superficie territorial de 140,000 km² que colinda en el este con el mar Caribe, en el oeste con el océano Pacifico, en el norte con Honduras y en el sur con Costa Rica. El territorio nacional está dividido políticamente en quince Departamentos y dos Regiones Autónomas, la Región Autónoma Costa Caribe Norte (RACCN) y la Región Autónoma Costa Caribe Sur (RACCS) que anteriormente conformaban el Departamento de Zelaya. Las regiones autónomas están localizadas en la llanura atlántica y cubren casi el 50\% del territorio nacional.

Nicaragua tiene un clima tropical y está localizada entre $11^{\circ}$ y $15^{\circ}$ de latitud norte. El clima del país consiste de una época lluviosa, de mayo a octubre, seguida por una época seca, de noviembre a abril. En la zona atlántica la lluvia es abundante y persistente; con una estación húmeda de 6-8 meses. La temperatura media anual varía entre los $28^{\circ} \mathrm{C}$ en las regiones costeras y llanuras, y los $20^{\circ} \mathrm{C}$ en las altas montañas del interior. La variación climática es más notable en el caso de la precipitación pluvial; ésta oscila entre los 700-800 mm de lluvia anual en las regiones más secas (que corresponde a un bosque xerofítico tropical), hasta los 4,000-5,000 $\mathrm{mm}$ anuales en las zonas más húmedas (donde predomina el bosque superhúmedo del tipo pluvioselva).

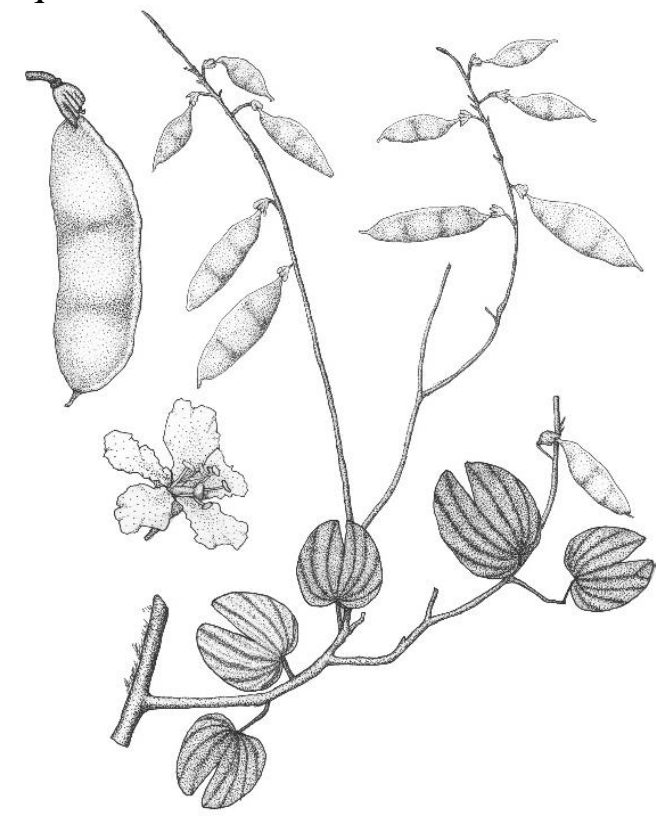

Fig. 2. Bauhinia herrerae. Dibujo de Felix Coe.

El país está conformado por tres regiones geomorfológicas: a) la Región del Pacífico. que consiste en una vertiente paralela a la costa litoral, que incluye el lago de Managua y el de Nicaragua. También presente en esta región están los volcanes Cerro Negro, Concepción, Masaya, Momotombo, San Cristóbal, Santiago y Telica. Esta región tiene las mejores tierras agrícolas, es la más densamente poblada y desarrollada del país. b) La Región Central es la de mayor altura con elevaciones de 1,500-2,000 metros sobre el nivel del mar. Es la región más montañosa con numerosas cumbres boscosas, mesetas, serranías y valles. En sus partes elevadas se encuentran asociaciones de pinos, robles, y bosques nublados con plantaciones de café. El área al este del lago de Nicaragua es de vocación ganadera. c) La Región Atlántica consiste en una llanura aluvial caracterizada por sus numerosos ríos. El extremo norte de la región atlántica (aproximadamente el 25\% del área) lo constituyen sabanas de pino caribe (Pinus caribaea), y el resto, mayormente bosques húmedos resultantes de la alta precipitación pluvial, especialmente en el extremo sur. La zona litoral se caracteriza por numerosos barreras litorales, deltas, lagunas costeras y pantanos. 


\section{AREA DEL ESTUDIO Y ECOSISTEMAS}

El territorio nicaragüense abarca alrededor de $140,000 \mathrm{~km}^{2}$. Las tierras bajas de la región atlántica de Nicaragua son las más extensas del país, constituyendo cerca de un tercio $(41,000$ $\mathrm{km}^{2}$ ) del territorio nacional. La elevación en esta área se extiende desde el nivel del mar hasta $200 \mathrm{~m}$. El clima es tropical, con una estación lluviosa de 6-8 meses y sin una estación seca bien definida. Los factores climáticos se combinan para producir una selva tropical (con una precipitación media anual de 3,810 mm), la más extensa en Centroamérica. Debido a la densidad de la pluvioselva, la abundancia de las lluvias y el gran número de ríos, la región atlántica de Nicaragua se mantuvo subdesarrollada y aislada del resto del país, hasta que recientemente se construyeron caminos de acceso. El principal medio de transporte en las tierras bajas de la región atlántica sigue siendo por barco o avión. La ciudad más grande es Bluefields con $60 \%$ de la población de las tierras bajas. La mayoría de los asentamientos de las etnias indígenas se encuentra en las áreas costeras o en las márgenes de lagunas y ríos. El tipo de bosque primario alrededor de la mayoría de asentamientos es el bosque pantanoso de tierras bajas (Sutton 1989), caracterizado por la abundancia de manglares y palmeras.

Para el propósito de este estudio se adoptó la división del país en tres regiones: la atlántica, norcentral y pacífica, de acuerdo a Stevens et al. (2004) en La Flora de Nicaragua. Este estudio se realizó en la región atlántica que incluye la Región Autónoma Costa Caribe Norte (RACCN), Región Autónoma Costa Caribe Sur (RACCS) y partes de los departamentos de Boaco, Chontales, Jinotega, Matagalpa y Río San Juan. La elevación puede llegar hasta $700 \mathrm{~m}$ sobre el nivel del mar en algunos picos aislados. La precipitación varía desde unos 1,700 $\mathrm{mm}$ alrededor de San Miguelito (Río San Juan) hasta unos 6,000 mm en San Juan del Norte (Río San Juan), obviamente con una fuerte gradiente este - oeste. La vegetación en esta área es muy diversa e incluye pluvioselva, bosque muy húmedo, bosque nublado o nebliselva, sabana de pino, pantanos y bosques de galería, manglares y playas (Incer 1975, Stevens et al. 2001). A continuación se describe los ecosistemas presentes en el área del estudio.

\section{Pluvioselva}

La pluvioselva está restringida a la esquina suroriental del país e incluye la parte oriental de Río San Juan y de Zelaya. Se caracteriza por una precipitación alta de 4,000-6,000 mm, esencialmente sin estación seca (ningún mes recibe menos de $200 \mathrm{~mm}$ ). La elevación es menos de los $100 \mathrm{~m}$ sobre el nivel del mar, pero con picos aislados que alcanzan los $300 \mathrm{~m}$. El bosque alto siempreverde consiste de cuatro doseles, bejucos y epífitas, de hábito herbáceo y leñoso. Este es el tipo de vegetación taxonómicamente el más diverso de Nicaragua y ninguna especie individual es la más dominante en el bosque inalterado. Algunas especies de árboles grandes son Andira inermis (almendro de río), Carapa guianensis (cedro macho), Dialium guianense (comenegro), Dipteryx oleifera (almendro), Laetia procera (plomo), Tabebuia guayacan (cortés), Virola koschnyi (banak colorado) y Vochysia guatemalensis (palo de agua). Las Rubiaceae y Melastomataceae son abundantes en el sotobosque. 


\section{Bosque muy húmedo}

La demarcación entre la pluvioselva y el bosque muy húmedo en la región atlántica es inexistente. El bosque muy húmedo es la vegetación nativa de la vertiente del Atlántico excepto donde está reemplazado por pluvioselva o sabana. La precipitación está comprendida entre los 2,000-4,000 mm, con una estación seca pronunciada de dos a cuatro meses. La elevación varía desde el nivel del mar hasta $700 \mathrm{~m}$. El bosque es principalmente siempreverde, aunque algunos árboles grandes son brevemente caducifolios. El bosque maduro generalmente tiene tres doseles y abundan tanto los bejucos como las epífitas. La diversidad es relativamente alta y rara vez se encuentra una especie de árbol dominante. Entre los árboles grandes conspicuos se encuentran Brosimum alicastrum (ojoche), Cedrela odorata (cedro real), Ceiba pentandra (ceiba), Guarea grandifolia (pronto alivio), Sciadodendron excelsum (lagarto), Sideroxylon capiri ssp. tempisque (tempisque) y Spondias mombin (jocote jobo).

\section{Bosque nublado o nebliselva}

El bosque nublado o nebliselva se encuentra en elevaciones superiores a las del bosque muy húmedo. Su distribución comienza por encima de los 600 metros sobre el nivel del mar. Hay una época seca marcada, pero como el nombre implica, estos bosques están a menudo cubiertos de una capa de nubes que ayuda a mantener una rica diversidad de epífitas. Las especies con los árboles más grandes pertenecen al género Quercus (roble-encino) Fagaceae y las Lauraceae. Otras especies asociadas con este ecosistema son de las familias Fabaceae, Flacourtiaceae, Hamamelidaceae, Juglandaceae, Meliaceae, Moraceae, Sabiaceae, Sapindaceae y Symplocaceae.

\section{Sabana de pino}

Las sabanas de pino se encuentran en forma de rodales dispersos, a veces extensos, a lo largo de la costa del Atlántico, desde la frontera con Honduras en el norte y en el sur hasta el Pinal, 20 $\mathrm{km}$ al sur de Laguna de Perlas. Los rodales a veces se encuentran atravesados por el bosque de galería e irregularmente se entremezclan con bosque húmedo siempreverde. La precipitación varía desde los 2,500-3,500 $\mathrm{mm}$ y los suelos son extremadamente pobres consistiendo de arena hasta grava. Las quemas son frecuentes y son un aspecto predominante de la ecología. El árbol dominante es Pinus caribaea var. hondurensis (pino), que puede formar rodales densos, pero existen áreas extensas frecuentemente inundadas y cubiertas de especies herbáceas de las familias Cyperaceae, Fabaceae y Poaceae. Las especies arbustivas más comunes son Byrsonima crassifolia (nancite) y Curatella americana (hoja-chiguie). Las áreas abiertas con poco drenaje están dominadas por las Cyperaceae, especialmente de los géneros Bulbostylis, Fimbristylis, Rhynchospora y Scleria. Mientras las Poaceae y los pinos se concentran en áreas con mejor drenaje y más arenosos. En los márgenes de este ecosistema existe una zona de transición hacia el bosque siempre verde dominada por arbustos de Melastomataceae y Rubiaceae.

\section{Pantanos y bosques de galería}

Los bosques de galería se encuentran adyacentes a los cauces de agua y son muy diferentes de la vegetación que los rodea, particularmente en las áreas sabaneras y los bosques caducifolios. Los bosques están sujetos a inundaciones frecuentes durante la época de lluvias y los suelos están saturados permanentemente. Los árboles predominantes en estas áreas pertenecen mayormente a los géneros Ficus (chilamate) e Inga (guava). En la región del Atlántico los 
árboles comunes en estas áreas son Heliocarpus appendiculatus (majagua), Hibiscus pernambucensis (majagua), Luehea seemannii (guácimo macho) y Ochroma pyramidale (balsa) y bejucos de los géneros Bauhinia (escalera de mico) y Mucuna (ojo de buey) casi siempre están presentes.

Los bosques pantanosos del Atlántico están en las tierras bajas costeras. El bosque está mayormente inundado y el suelo siempre saturado. Arboles conspicuos son Bactris gasipaes (pejibaye), Bravaisia integerrima (mangle blanco), Ceiba pentandra (ceiba), Erythrina fusca (elequeme), Pachira aquatica (poponjoche), Raphia taedigera (jolillo) y Symphonia globulifera (leche maría). Ciertas áreas pantanosas están dominadas por especies herbáceas, en particular Cyperaceae y Poaceae.

\section{Manglares}

En toda América tropical y en ambas costas de Nicaragua, los manglares son generalmente similares, tanto en estructura como en la composición de especies. Los límites de los manglares están definidos por sumersión periódica en agua salada debido a las mareas. Los árboles que se encuentran en los manglares están muy adaptados y restringidos a este ambiente. Estos bosques han sido estudiados extensamente y son comunidades económicamente importantes. La diversidad es baja y las especies más comunes de árboles son Avicennia germinans (mangle

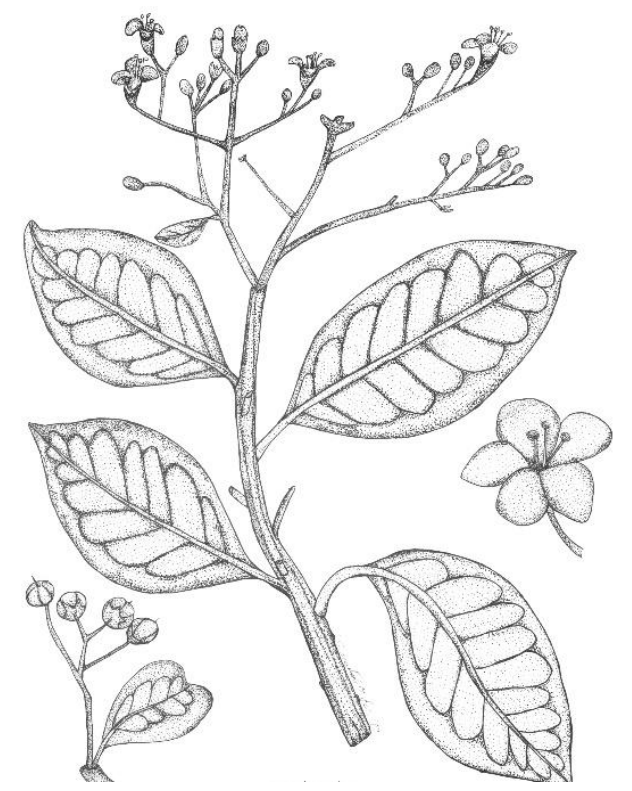
negro), Conocarpus erectus (botoncillo), Laguncularia racemosa (mangle blanco) y Rhizophora mangle (mangle colorado).

\section{Playas}

La vegetación de las playas (lagunas y marinas) son típicas de las playas de la América tropical. Muchas especies solo se encuentran en estas áreas debido a la salinidad y a perturbaciones. Las plantas herbáceas comunes que forman tapetes en las playas arenosas se encuentran Canavalia rosea (frijol de playa), Galactia striata (frijolillo), Ipomoea pes-caprae (camote de playa) y Sphagneticola trilobata (margarita rastrera). Entre los árboles más grandes se encuentran Chrysobalanus icaco (icaco), Coccoloba uvifera (uva de playa), Cocos nucifera (coco), Tamarindus indica (tamarindo) y Terminalia cattapa (almendra).

Fig. 3. Bourreria succulenta. Dibujo de Felix Coe. 


\section{METODOLOGÍA}

Este estudio se ejecutó siguiendo los pasos siguientes: (a) la recopilación de la bibliografía relevante a los estudios botánicos en Nicaragua; (b) el estudio de ejemplares de herbario para la obtención de datos etnobotánicos, florístico, ecológicos y taxonómico; (c) el trabajo de campo; (d) la identificación de ejemplares botánicos, y (e) el trabajo de laboratorio (análisis químico y bioensayos de los ejemplares botánicos).

(b) Para complementar los datos etnobotánicos, ecológicos, florísticos y taxonómicos obtenidos por el autor fueron revisadas las etiquetas de especímenes colectados por otros botánicos en la región atlántica de Nicaragua que están depositados en los siguientes herbarios: el New York Botanical Garden (NYBG), Bronx, NY, USA; el Missouri Botanical Garden (MO), Saint Louis, MO, USA; el Harvard University Herbaria (GH), Boston, MA, USA; el Herbario Nacional de Honduras (TGCH-UNH) en la Universidad Nacional Autónoma de Honduras, Tegucigalpa, Honduras; en al Herbario de la Escuela Agrícola Panamericana (EAP), El Zamorano, Honduras; el Herbario Nacional de Nicaragua (HNMN) en la Universidad Centro Americana, Managua; y el Museo Nacional de Costa Rica, San José, Costa Rica.

(c) El trabajo de campo consistió en entrevistas y encuestas de los individuos involucrados en la prestación de salud (curanderos y comadronas), en actividades de subsistencia y con otros miembros de las comunidades. Las metodologías empleadas fueron similares a las utilizadas por Chazdon y Coe (1999), Coe (1994), y Coe y Anderson (1996a, b, 1997, 1999). Los estudios de campo se realizaron durante más de cuarenta años, pero se intensificaron de 1990 hasta 2000 por el autor, que es nativo de Bluefields y ha estado estudiando la etnobotánica de esta región desde 1972. La mayoría de las entrevistas se llevaron a cabo en inglés y español, sin embargo, cuando era necesario se utilizó un intérprete para las lenguas indígenas (miskito, sumu, rama y garífuna). El trabajo de campo se realizó en dos fases: (a) la recopilación de información a través de encuestas, notas de campo y entrevistas en grabaciones de audio-casetes para documentar los usos de las plantas y para compilar una lista de las especies, y (b) giras al campo para colectar especímenes botánicos. Este estudio se llevó a cabo en la región atlántica, lo cual se describió anteriormente en la sección de Área del Estudio y Ecosistemas.

(d) La identificación y clasificación de las plantas se basaron en las siguientes fuentes: (a) Ferns and their Allies (Davidse et al 1995), (b) las gimnospermas (Stevens et al 2001), (c) las angiospermas (Cronquist 1981; Stevens et al., 2001, (d) los nombres de especies (Stevens et al 2001), (e) abreviaturas del nombre del autor (Brummitt y Powell 1992), (f) abreviaturas de libros (Stafleu y Cowan1976-1988; Stafleu y Mennega 1992-2000), (g) abreviaturas de revistas (Bridson 1991), (h) nombres de localidades (CIDCA 1986,1987,1989; Conzemius 1927,1932; Guerrero y Guerrero 1985; Incer 1985; Smutko 1985), y (i) los nombres vernáculos o comunes de las plantas (CIDCA 1986 , 1987,1989; Conzemius1927, 1932; Smutko 1985 y las obtenidas por el autor).

Especímenes botánicos fueron depositados en el Herbario Nacional Managua, Nicaragua (HNMN), el Missouri Botanical Garden (MO) y la Universidad de Connecticut (CONN). La mayoría de los ejemplares botánicos fueron identificados en la Universidad de Connecticut, 
Storrs, CT y el Missouri Botanical Garden, St. Louis, MO. Algunos especímenes botánicos fueron enviados a especialistas en el Missouri Botanical Garden (MO) y el New York Botanical Garden (NY) para ser identificados. Los nombres de localidades y nombres comunes de plantas se verificaron en la medida de lo posible utilizando fuentes publicadas (Duke 1972, Hadel 1975, Howes 1974, Guerrero y Soriano de Guerrero 1985, Incer 1985, Martínez 1991, Morton 1981, Uphof 1968).

e) En este trabajo, las actividades biológicas y los compuestos dados para cada especie fueron obtenidos por medio de búsqueda bibliográfica de los principales compendios (publicados entre 1958 y 1994), para determinar la presencia o ausencia de alcaloides o glucósidos en las especies medicinales. Las búsquedas electrónicas se realizaron usando Chemical Abstracts Services (1958-1994), PubMed, Web of Science, Scielo, la Universidad de Illinois en Chicago NAPRALERT (acrónimo de "Natural products Alert") y publicaciones en numerosas revistas científicas. Las especies cuya química no fue encontrada en la literatura fueron ensayadas en mi laboratorio, para determinar la presencia de alcaloide usando el reactivo de Dragendorff (Bums 1964, Harbome 1988, Raffauf 1962, Stahl 1969, Stermitz et al., 1989) y las técnicas establecidas por Schultes y Raffauf (1990). Los ensayos biológicos fueron hechos en el laboratorio del autor (Coe 1994; Coe y Anderson 1996a, 1996b, 1997, 1999, 2005; Coe 2008a, 2008b; Coe, Parikh and Johnson 2010; Coe et al. 2012). Los nombres de compuestos orgánicos se verificaron usando el Diccionario de Compuestos Orgánicos (Cook, Bunbury y Hey 1965).

\section{ETNOBOTÁNICA Y LOS DERECHOS DE PROPIEDAD INTELECTUAL}

En el contexto mundial, el saber tradicional de los pueblos indígenas incluye sus creencias, artes, espiritualidad y otras formas de experiencia y expresión cultural. Desde la adopción de la Declaración Universal de Derechos Humanos en 1948, la propiedad intelectual ha sido considerada como un derecho humano fundamental de todos los pueblos indígenas. Sin embargo, recientemente, la necesidad de proteger, preservar y proveer para el uso justo de la propiedad intelectual indígena -el saber tradicional- entró en el debate nacional e internacional sobre los derechos de propiedad intelectual. Una preocupación particular para los pueblos indígenas es como un derecho humano fundamental de todos los pueblos indígenas. Sin embargo, recientemente, la necesidad de proteger, preservar y proveer para el uso justo de la propiedad intelectual indígena -el saber tradicional-.

El saber tradicional sobre las plantas nativas de la Región Atlántica de Nicaragua (RAN) podría ser descrito como parte del patrimonio de los pueblos indígenas de esta región. Sin embargo, como en muchas partes del mundo, este derecho no está protegido actualmente por la ley nicaragüense. Para salvaguardar los intereses de los pueblos indígenas de la RAN es necesario desarrollar acuerdos de investigación entre los investigadores y las comunidades indígenas. Es vital establecer una relación o asociación basada en confianza y mutuo respeto. El acuerdo debe asegurar que ambas partes reciban una distribución equitativa por cualquier beneficio que pudiera derivarse de la comercialización de los resultados de la investigación.

Es mi opinión, toda la información etnobotánica que me han proporcionado a lo largo de las décadas el pueblo de la RAN debe estar libremente disponible para uso público. Este conocimiento es de dominio público y no debe usarse para beneficio personal o en una agenda 
política. La mayoría de los pueblos indígenas involucrados en este estudio no están conscientes del concepto de Derechos de Propiedad Intelectual. Uno de mis objetivos inmediatos es ayudar a educar a los indígenas de la RAN sobre este tema con la esperanza de salvaguardar esta parte de su patrimonio. Propongo que todos los beneficios derivados de estudios que involucren a los indígenas de la RAN se inviertan en sus comunidades. Por ejemplo, todos los ingresos de la venta de este libro y futuros libros que publico sobre temas basados en el saber indígena de la RAN serán otorgados a la Fundación para la Autonomía y Desarrollo de la Costa Atlántica de Nicaragua (FADCANIC), para ser invertidos en las comunidades indígenas. Me gustaría ver, primero, una inversión en programas que ayuden a los practicantes de la medicina tradicional en mantener y promover el uso de la medicina ancestral, y segundo, incrementar la interacción entre la medicina tradicional y la moderna. Espero que mis investigaciones y esta obra ayuden a promover un renacimiento en el interés de la medicina tradicional y quizás ayude a reavivar el respeto e interés por el saber etnomedicinal en las comunidades de la RAN. En una perspectiva general quizás sirva como un catalizador para fomentar un interés renovado en la cultura tradicional de los pueblos indígenas de la RAN.

\section{RESULTADOS Y DISCUSION}

En la Flora de Nicaragua se describe 5,796 especies en 1,699 géneros en 225 familias (Stevens et al., 2004). De ellas, se estima que 3,000-3,500 especies ocurren en región atlántica de Nicaragua (RAN). La flora medicinal documentada en este estudio consiste de 852 especies en géneros y en familias. Basados en los estudios del autor, la etnofarmacopea de los grupos indígenas de esta región alcanza más de 600 especies de plantas vasculares (Coe 1994; Coe y Anderson 1996a, 1996b, 1997, 1999, 2005; Coe 2008a, 2008b; Coe, Parikh and Johnson 2010; Coe et al. 2012). Las 252 especies restantes son usadas por grupos indígenas en otras áreas de Nicaragua u otros países; pero son incluidas porque proveen especies alternativas que pueden ser usadas por practicantes de la medicina tradicional. Creo que es importante incluirlas porque muchas de las especies que fueron antiguamente usadas ya no se encuentran debido a la deforestación en muchas de las áreas de región atlántica de Nicaragua.

Este trabajo documenta la flora medicinal y la farmacología de las especies de la RAN. Es el resultado de cientos de años de recolección del saber etnomedicinal de las etnias de esta región y más de cuarenta años de estudio por parte del autor. Para mayor información acerca de estos estudios ver las referencias bibliográficas al final del libro. Obviamente, el conocimiento sobre la flora medicinal del atlántico nicaragüense continuará acumulándose. Espero que este trabajo sirva de base y estímulo para estudios futuros de la flora medicinal y la etnofarmacología de la RAN.

En el transcurso de mis estudios en la RAN, más de 600 especies de plantas fueron sometidas a pruebas de alcaloides, y de çestas, el $69 \%$ resultó positivo. Este porcentaje se compara con un $16 \%$ de plantas ricas en alcaloides en un estudio de Hazlett \& Sawyer (1998). Este estudio incluyo el $80 \%$ de las plantas en un área geográfica particular, la estepa de pastos cortos (Hazlett \& Sawyer 1998). Además, indicaron que cuando un gran porcentaje de todas las especies de plantas de una zona son probadas, los porcentajes de plantas ricas en alcaloides tienden a estar entre 15-20\%. El mayor porcentaje de plantas ricas en alcaloides en mis estudios es debido probablemente porque las plantas probadas no fueron seleccionadas al azar, sino que forman 
parte de la etnofarmacología. Estas plantas empíricamente seleccionadas aparentemente se utilizaron, en parte, porque contienen alcaloides bioquímicamente ricos.

Los indígenas de la RAN obtienen más del $80 \%$ de las especies medicinales del bosque secundario (ver Tabla 1). Esta preferencia es debido al acceso a estos bosques y la abundancia de especies con compuestos bioactivos (Kohn 1992; Coe 1994; Coe y Anderson 1996a; Voeks 1996; Coe y Anderson 1997, 1999; Coe 2008a, 2008b). Desde el punto de vista utilitario, el bosque secundario provee la mayoría del material botánico que requieren los grupos indígenas para su subsistencia diaria. Estos resultados son similares a los obtenidos en el noreste de Costa Rica por Chazdon \& Coe (1999). En un futuro no muy lejano el bosque secundario en la RAN se convertirá en la fuente principal de productos maderables y no maderables para los grupos indígenas. Esto está basado en los resultados del estudio de Chazdon \& Coe (1999) que demostró que el bosque secundario bien desarrollado tiene una abundancia bastante alta de especies medicinales. Otro factor importante es que la mayoría de las especies del bosque secundario es de uso múltiple, particularmente las especies arbóreas. Aunque la mayoría de las especies medicinales son herbáceas, las especies arbóreas son las más utilitarias.

Tabla 1. Especies medicinales nativas y su hábitat de procedencia.

\begin{tabular}{|c|c|c|c|c|}
\hline Etnia & \multicolumn{2}{|c|}{$\begin{array}{l}\text { Bosque } \\
\text { Secundario }\end{array}$} & $\begin{array}{l}\text { Bosque } \\
\text { Primario }\end{array}$ & Total \\
\hline Miskitu & $86 \%$ & 14 & & 290 \\
\hline Garífuna & $84 \%$ & 16 & & 214 \\
\hline Rama & $81 \%$ & 19 & & 190 \\
\hline Sumu & $86 \%$ & 14 & & 187 \\
\hline
\end{tabular}

De las 852 especies en este compendio es difícil seleccionar cuál de ellas, si existe alguna, será importante con respecto al uso humano. No obstante he seleccionado diez especies que, por razones objetivas y subjetivas, resultan más dignas de futuras investigaciones, éstas son: Acisanthera quadrata, Alibertia edulis, Chrysothemis pulchella, Eugenia venezuelensis, Hyptis verticillata, Lygodium heterodoxum, Olyra latifolia, Petiveria alliacea, Struthanthus cassythoides and Vovhysia ferruginea. En los ensayos químicos y citotóxicos de estas especies resultaron positivas para alcaloides y a la vez tuvieron alta toxicidad. Las pruebas de alcaloides de las especies medicinales usadas por los grupos indígenas de la RAN resultaron con un promedio de $69 \%$ positivas y $8 \%$ para los glucósidos (ver Tabla 2).

Tabla 2. Especies medicinales usados por los grupos indígenas y su bioactividad.

\begin{tabular}{|l|c|c|c|c|c|}
\hline Etnia & Especies & Genero & Familia & Alcaloide & Glucósido \\
\hline Miskitu & 310 & 234 & 85 & $59 \%$ & $5 \%$ \\
\hline Garífuna & 229 & 175 & 71 & $62 \%$ & $5 \%$ \\
\hline Rama & 190 & 144 & $70 \mathrm{i}$ & $68 \%$ & $11 \%$ \\
\hline Sumu & 187 & 146 & 69 & $87 \%$ & $13 \%$ \\
\hline \multicolumn{7}{|l|l|}{} \\
\hline
\end{tabular}


La flora de la RAN es un de las más diversas de Centroamérica. Antes de la publicación de la Flora de Nicaragua, la flora nicaragüense era la más pobremente estudiada de los países centroamericanos. Esto es irónico considerando que es la más diversa de la región. La flora medicinal de la región atlántica todavía necesita más estudios botánicos y fitoquímicos para determinar su potencial farmacéutico tanto de las especies conocidas y las que aún no han sido descubiertas. Como se sabe la deforestación y por consiguiente la desaparición de hábitat tiene como consecuencia la extinción de especies lo cual es un problema a nivel mundial. Con esto en mente se debe tratar de proteger los ecosistemas de la vertiente atlántica de Nicaragua. Con el avance de la frontera agrícola en esta área en las últimas décadas, la deforestación ha causado daños significativos a los ecosistemas, muchas de ellos frágiles y vitales para mantener el balance ecológico y la biodiversidad del área. Las especies comunes son relativamente bien conocidas y abundantes, pero hay especies raras (e.g., Aristolochia trilobata, Cissampelos pareira, Dorstenia contrajerva, Fevillea cordifolia, Quassia amara) que están en peligro de desaparecer de esta región debido a la destrucción de hábitat. Esta situación debería ser una alarma para todos. Con el fin de reducir las extinciones futuras, Nicaragua necesita proteger vigorosamente las áreas naturales que aún quedan, tanto para mantener su patrimonio de diversidad, como para proteger su clima futuro. Adicionalmente, deberían hacerse consideraciones serias de cuáles componentes de su diversidad no están actualmente protegidos en el sistema nacional de reservas.

Los detalles sobre información de laboratorio y literatura comprenden la mayor parte de este libro. Para facilitar las identificaciones, cada especie también tiene un bosquejo botánico. Las especies incluidas en esta obra están arregladas en orden alfabético por sus nombres científicos; y los nombres científicos, familias y el sistema de clasificación están de acuerdo a La Flora de Nicaragua (Stevens et al. 2001). Para cada especie se incluye la siguiente información: nombre científico, sinónimo, familia, nombre común [en español (h), inglés estándar (e), ingles criollo (c), miskitu $(\mathrm{m})$; sumu/ulwa (u); rama (r) y garífuna (g)], descripción, hábitat y distribución, propiedades y acciones, usos medicinales, composición química, y actividad biológica. En la información de la especie se incluye frecuencia con que se encuentra la especie en el país; hábito o tipo de vegetación; la zona natural o departamento; la elevación donde crece; la época de floración (fl) y fructificación (fr); los meses del año -abreviado con las tres primeras letras; se citan cinco colecciones representativas del área del estudio con el apellido del colector y su número de colección; y la distribución de las especies. Los nombres comunes o vernáculos dados son los obtenidos en el campo o de otras fuentes por el autor.

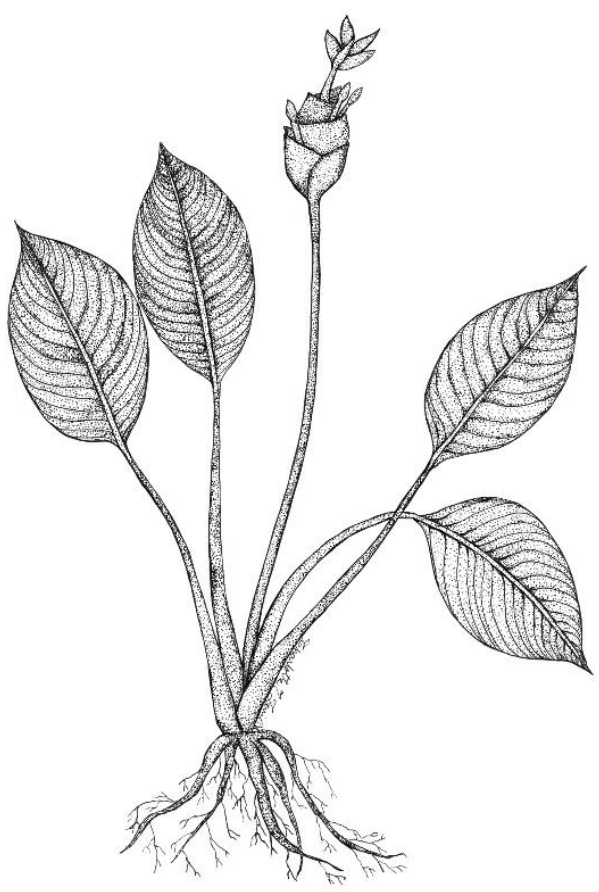

Fig. 4. Calathea macrosepala Coe. Dibujo de Felix Coe. 


\section{REFERENCIAS BIOGRAFICAS}

Barrett B. 1994. Medicinal plants of Nicaragua's Atlantic coast. Economic Botany 48(1):8-20.

Canter PH, Thomas H, Ernst E. 2005. Bringing medicinal plants into cultivation, opportunities and challenges for biotechnology. Trends in Biotechnology 23(4):180-185.

Cochrane CB, Nair PK, Melnick SJ, Resek AP, Ramachandran C. 2008. Anticancer effects of Annona glabra plant extracts in human leukemia cell lines. International Institute of Anticancer Research 28(2A):965-71.

Coe FG, Anderson GJ. 1996a. Ethnobotany of the Garifuna of eastern Nicaragua. Economic Botany 50(1):71-107.

Coe FG, Anderson GJ. 1996. b Screening of medicinal plants used by the Garifuna of Eastern Nicaragua for bioactive compounds. Journal of Ethnopharmacology 53(1):29-50.

Coe FG, Anderson GJ. 1997. Ethnobotany of the Miskitu of eastern Nicaragua. Journal of Ethnobiology 17:171-214.

Coe FG, Anderson GJ. 1999. Ethnobotany of the Sumu (Ulwa) of Southeastern Nicaragua and Comparisons with Miskitu Plant Lore. Economic botany 53(4):363-386.

Coe FG, Anderson GJ. 2005. Snakebite ethnopharmacopoeia of eastern Nicaragua. Journal of Ethnopharmacology 96(1):303-23.

Coe FG, Parikh DM, Johnson CA, Anderson GJ. 2012. The good and the bad: Alkaloid screening and brineshrimp bioassays of aqueous extracts of 31 medicinal plants of eastern Nicaragua. Pharmaceutical Biology 50(3):384-92.

Coe FG, Parikh DM, Johnson CA. 2010. Alkaloid presence and brine shrimp (Artemia salina) bioassay of medicinal species of eastern Nicaragua. Pharmaceutical Biology 48(4):439-45.

Coe FG. 1994. Ethnobotany of the Garifuna of Eastern Nicaragua. Ph.D. Dissertation (Botany), University of Connecticut, Storrs.

Coe FG. 2008a. Ethnobotany of the Rama of southeastern Nicaragua and comparisons with Miskitu plant lore1. Economic Botany 62(1):40-59.

Coe FG. 2008b. Ethnomedicine of the Rama of southeastern Nicaragua. Journal of Ethnobiology 28(1):1-38.

Coe FG. 2008c. Rama midwifery in eastern Nicaragua. Journal of ethnopharmacology 117(1):136-57.

Davidse G, Sousa M. 2009. Flora Mesoamericana Volume 4, Part 1.

Davidse G, Sousa SM, Chater AO, Rico-Arse M. 1995. Flora Mesoamericana. Alismataceae a Cyperaceae. Botanische Jahrbucher 117(3):378

Davidse G, Ulloa C. 2012. Flora Mesoamericana Volume 4, Part 2.

Davidse G, Ulloa C. 2015. Flora Mesoamericana Volume 2, Part 3.

Davidse, G, Sousa M, Knapp S, Moran C, Riba R, Chiang F, Barrie F. 1995. Flora Mesoamericana Volume 1, Instituto de Biología, Universidad Nacional Autónoma de México, México, DF.

Davidson WV. 1980. The Garífuna of Pearl Lagoon: Ethnohistory of an afro-american enclave in Nicaragua. Ethnohistory pp.31-47.

Dennis PA, Olien MD. 1984. Kingship among the Miskito. American Ethnologist 11(4):718737. 
Dennis PA. 1981. Part three: Grisi Siknis Among the Miskito. Medical anthropology 5(4):445505.

Dennis PA. 1988. Herbal medicine among the Miskito of Eastern Nicaragua. Economic Botany 42(1):16-28.

Helms MW. 1971. Asang: adaptations to culture contact in a Miskito community. University of Florida Press.

Helms MW. 1983. Miskito slaving and culture contact: ethnicity and opportunity in an expanding population. Journal of Anthropological Research 39(2):179-197.

Lopez JA, Saenz JA, Slatkin DJ, Knapp JE, Schiff PL. 1976. Flavonoid and lipid constituents of Hymenaea courbaril and Physalis angulata. Phytochemistry 15(12):2028.

Loveland FO. 1976a. Dialectical Aspects of Natural Symbols: Order and Disorder in Rama Indian Cosmology.

Loveland FO. 1976b. Snakebite cure among the Rama Indians of Nicaragua. Medical anthropology pp.81-102.

Loveland FO. 1976b. Tapirs and manatees: cosmological categories and social process among Rama Indians of eastern Nicaragua. Philadelphia Institute for the Study of Human Issues, Philadelphia, PA pp.67-82.

Loveland FO. 1982. Watch that pot or the Waksuk will eat you up: an analysis of male and female roles in Rama Indian myth. Sex roles and social change in native lower Central American societies, eds. F. Loveland and CA Loveland pp.124-141.

Nietschmann B. 1969. The distribution of Miskito, Sumu, and Rama Indians, Eastern Nicaragua. International Union of Anthropological and Ethnological Sciences

Nietschmann B. 1972. Hunting and fishing focus among the Miskito Indians, eastern Nicaragua. Human Ecology 1(1):41-67.

Nietschmann B. 1973. Between land and water: the subsistence ecology of the Miskito Indians, Eastern Nicaragua. Seminar Press.

Nietschmann B. 1979. Ecological change, inflation, and migration in the far western Caribbean. Geographical review pp.1-24.

Nietschmann B. 1990. Conservation by conflict in Nicaragua. Natural history (USA).

Stevens WD, Ulloa C, Pool A, Montiel OM, eds. 2001. Flora de Nicaragua. Tomo I-III. Missouri Botanical Garden Press, St. Louis. 